This item was submitted to Loughborough's Research Repository by the author.

Items in Figshare are protected by copyright, with all rights reserved, unless otherwise indicated.

\title{
A new empirical 'exponential' tyre model
}

\section{PLEASE CITE THE PUBLISHED VERSION}

http://dx.doi.org/10.1504/IJVD.2014.060778

\section{PUBLISHER}

(C) Inderscience Enterprises Ltd.

\section{VERSION}

AM (Accepted Manuscript)

\section{PUBLISHER STATEMENT}

This work is made available according to the conditions of the Creative Commons Attribution-NonCommercialNoDerivatives 4.0 International (CC BY-NC-ND 4.0) licence. Full details of this licence are available at: https://creativecommons.org/licenses/by-nc-nd/4.0/

\section{LICENCE}

CC BY-NC-ND 4.0

\section{REPOSITORY RECORD}

Best, Matt C.. 2015. “A New Empirical 'exponential' Tyre Model”. figshare. https://hdl.handle.net/2134/17362. 


\title{
A New Empirical ‘Exponential’ Tyre Model
}

\author{
Matthew C Best \\ Senior Lecturer in Vehicle Dynamics and Control \\ Department of Aeronautical and Automotive Engineering \\ Loughborough University \\ Leicestershire \\ LE11 3TU, UK \\ E-mail: M.C.Best@lboro.ac.uk \\ Tel: +44 1509227209 \\ Fax: +44 1509227275
}

\begin{abstract}
In this paper a new and simple formula is presented for empirical modelling of tyre force data. Based on exponential functions, it is capable of matching single slip data for lateral or longitudinal force using three parameters which can be computed in terms of stiffness, peak and saturated force values. Through a factorial study, the three parameters are also reformulated into functions of load and slip to provide full mapping of $F_{x}$ and $F_{y}$ across the range of longitudinal slip, lateral slip and vertical load. Significantly, the resulting model does not rely on a total slip calculation, so it retains a simple structure in force vs slip or load derivatives. The new model is compared with two alternative simple tyre models, and is shown to map forces generated from a reference Pacejka model. It is also used to fit measured tyre force data accurately.
\end{abstract}

\section{Keywords}

tyre model; tire model; empirical model; vehicle dynamics; vehicle control

Biographical notes: Matt Best is a senior lecturer in vehicle dynamics and control in the department of Aeronautical and Automotive Engineering at Loughborough University in the UK. He gained his $\mathrm{PhD}$ in practical implementation of vehicle ride control at Loughborough in 1995, and has been a lecturer since 1996. His principal research interests include design of nonlinear optimal observers and controllers for vehicle ride and handling, Kalman filter methods for estimation and identification, vehicle dynamics modelling and real-time vehicle motion simulators. He has published approx. 40 papers in his field, in conferences, and journals such as International Journal of Vehicle Autonomous Systems, Proc. ImechE parts D and K, Vehicle System Dynamics, and International Journal of Vehicle Design.

\section{Introduction}

In modern chassis control systems, increasing use is made of the ever expanding computational capacity in the vehicle. As a result there is more use of model based real-time controllers (eg Andreasson \& Bunte 2006, Poussot-Vassal et al 2008) and observers (eg Doumiati et al 2011). In both on and off-line applications the vehicle model depends heavily on the tyre contact model, which must deliver a suitably accurate force prediction, and although processor speed and capacity is increasing, any on-line tyre model must also remain 
computationally efficient. A range of more complex models exist, catering eg for camber variation, self-aligning moment, but the majority of applications have the core requirement of predicting lateral and longitudinal tyre forces as a function of vertical load, lateral slip $\alpha$ and longitudinal slip, $S$.

Most existing tyre models use a combined slip formulation whereby the forces are calculated as an (usually complicated) algebraic function of total slip $k$, defined as $\sqrt{\alpha^{2}+S^{2}}$ or similar. Most applications employ a simple form of Pacejka's magic tyre formula, but total slip is still fundamental to the few that do not (eg the Dugoff model in Doumiati et al 2011 or Burckhardt model in Li et al 2008). The most recent Pacejka tyre model (see Pacejka 2002) does not rely on total slip, but still requires formulae with embedded trigonometrical functions of functions. This model, along with those that use total slip, present quite a complicated model for the tyre forces, but they certainly result in extremely protracted functions for the derivatives of the forces, $\partial F_{y} / \partial \alpha, \partial F_{x} / \partial \alpha$ etc; these derivatives are required in some controller designs (eg Gordon \& Best 2006) and in extended (nonlinear) Kalman filter observers, which have attracted a great deal of interest as a key component in model based integrated chassis control designs (eg Wenzel et al 2006 and Kim 2009).

This paper proposes a new tyre model which fills this particular niche application, and will also have wider appeal due to its simplicity. The Exponential tyre model provides consistent, accurate longitudinal and lateral force prediction in a simple algebraic form which remains simple in all its derivatives, and relies on just 10 parameters. This is the first full description of the model; an embryonic first draft of the concept, which used 20 parameters, was introduced in Best (2010).

The basis of the model, described in Section 2, has two exponential terms and three parameters, and is similar to Pacejka's basic magic formula in that it reconstructs the essential 1-D force vs slip curve using non-physical parameters. Section 3 goes on to define three subfunctions which extend the exponential model to two dimensions and illustrates its fit to a reference, Pacejka model. The paper then illustrates and quantifies the computational advantages of the new model before presenting a case study reconstruction of a full combined slip model from tyre forces measured at the Calspan tyre test facility.

\section{Single Slip Definition and Prescription}

The basis of the Exponential tyre model is the expression :

$$
\bar{F}_{y}=A|\alpha| e^{-b|\alpha|}+B\left(1-e^{-b|\alpha|}\right)
$$

where $\bar{F}_{y}$ is lateral force normalised by friction and vertical load, $F_{y}=\mu F_{z} \bar{F}_{y} \operatorname{sgn}(\alpha)$ and $\alpha$ refers to lateral slip in the standard form, $\alpha=-v / u$ with $v$ and $u$ lateral and longitudinal contact patch velocities respectively. $\mu$ is considered a constant throughout this paper, but 
could be expanded as a function of vertical load in future without compromising the advantages of the model. The equivalent equation

$$
\bar{F}_{x}=A|S| e^{-b|S|}+B\left(1-e^{-b|S|}\right)
$$

applies for longitudinal slip $S=(r \omega-u) / u$ with $r \omega$ as rolling radius multiplied by wheel spin speed.

The form of the model arose from experimentation with simple algebraic functions to achieve the appropriate peak and lower constant force at high slip which is seen in experimental tyre data. Figure 1 illustrates how the linear and decaying exponential terms combine to form the peak, before smooth transition to the second exponential term which defines the terminal force. Lower values for $A$ and $b$ remove the initial peak and deliver the characteristic form of tyre model seen when cross slip is non-zero. Here, and later, 'cross slip' refers to $S$ in models for $F_{y}$ and $\alpha$ in models for $F_{x}$.

\section{** INSERT FIGURE 1 **}

The principal aim of the model is to map the tyre forces in a computationally efficient way, using a minimum number of parameters; as such, $A, B$ and $b$ are non-physical parameters which will generally need to be fitted to tyre data, or an alternative tyre model. However it is possible to rearrange eqns (1), (2), in order to prescribe $A, B$ and $b$ in terms of desired cornering or slip stiffness, $C_{\alpha}$ or $C_{x}$, peak force $F_{p}$ and the terminal force $F_{\text {term }}$, which $F_{y}$ settles to at high slip. Adopting the lateral force case with positive $\alpha$, and normalising the known values to $\bar{C}_{\alpha}=C_{\alpha} /\left(\mu F_{z}\right), \bar{F}_{\text {term }}=F_{\text {term }} /\left(\mu F_{z}\right)$ and $\bar{F}_{p}=F_{p} /\left(\mu F_{z}\right)$, for high slip the exponential terms in eqn (1) reduce to zero, so

$$
\bar{F}_{\text {term }}=B
$$

Differentiating, we can derive expressions for initial slope,

$$
\bar{C}_{\alpha}=\left.\frac{d \bar{F}_{y}}{d \alpha}\right|_{\alpha=0}=A+B b
$$

and peak slip $\alpha_{p}$ is obtained by solving $d \bar{F}_{y} / d \alpha=0$

$$
\alpha_{p}=\frac{A+B b}{A b}
$$

Substituting in $\bar{F}_{y}$, peak force is then

$$
\bar{F}_{p}=\frac{A}{b} e^{-(1+B b / A)}+B
$$

which can be rearranged in the form of the Lambert $\mathrm{W}$ function, $W e^{W}=X$ : 


$$
\frac{B b}{A} e^{B b / A}=\frac{B e^{-1}}{\bar{F}_{p}-B}
$$

Since $X$ is known, $W$ can be found using Matlab, with lambertw $(\odot, X)$. Alternatively, by Newton's method, recursive execution of

$$
W_{j+1}=W_{j}+\frac{X-W_{j} e^{W_{j}}}{e^{W_{j}}+W_{j} e^{W_{j}}}
$$

converges from $W_{0}=0$ in fewer than 10 iterations. Given that $W=B b / A$, substitution in eqn (4) provides $A$ and $b$ as,

$$
\begin{gathered}
A=\bar{C}_{\alpha} /(1+W) \\
b=A W / B
\end{gathered}
$$

A general reference model is used for fitting parameters in Section 2, and this is introduced here to provide realistic initial single force / slip comparison plots. The reference is a combined slip form of Pacejka's formula which uses similarity functions from Milliken \& Milliken, 2005 and Pacejka 2002, and has been used previously in Gordon \& Best 2006. Arguably it represents the simplest alternative tyre model to the proposed experimental model, and is defined

$$
\begin{aligned}
& C_{\alpha}=c_{1} F_{z r}\left(1-e^{c_{2} F_{z} / F_{z r}}\right) \\
& F_{p}=\mu F_{z} \min \left(1.6,\left(\frac{4 F_{z}}{F_{z r}}\right)^{-0.15}\right) \\
& \bar{S}=\frac{C_{\alpha} S}{\eta_{0} F_{p}} \quad \bar{\alpha}=\frac{C_{\alpha} \alpha}{F_{p}} \\
& k=\sqrt{\bar{S}^{2}+\bar{\alpha}^{2}} \\
& F_{r}=\sin \left(C \tan ^{-1}\left(\frac{k}{C}-E\left(\frac{k}{C}-\tan ^{-1}\left(\frac{k}{C}\right)\right)\right)\right) \\
& \eta_{1}=1 / 2\left(1+\eta_{0}\right)-1 / 2\left(1-\eta_{0}\right) \cos (k / 2), \quad k<2 \pi \\
& \eta_{1}=1 \\
& k \geq 2 \pi \\
& \rho=\frac{\eta_{1} \bar{\alpha}}{\eta_{0} \bar{S}} \\
& \bar{F}_{x}=\frac{\operatorname{sgn}(S) F_{r}}{\sqrt{1+\rho^{2}}} \\
& \bar{F}_{y}=\rho \bar{F}_{x}
\end{aligned}
$$




$$
F_{x}=F_{p} \bar{F}_{x} \quad F_{y}=F_{p} \bar{F}_{y}
$$

The parameters used here, for a nominal C class passenger car tyre, are given in Table 1.

** INSERT TABLE 1 **

To illustrate the capability of the basic exponential model, Figure 2 shows the results of four separate Nelder-Mead optimisations of $A, B$ and $b$ to fit four reference cases of lateral force vs slip. These were generated by evaluating the reference model at two typical vertical load and cross slip settings. A high accuracy of fit is achieved in all cases.

\section{** INSERT FIGURE $2 * *$}

\section{Extension to a Combined Slip Exponential Model}

The fitting process used to match reference data in Figure 2 provides a means of extending the Exponential model by considering the variations in $A, B$ and $b$ required to fit a range of load and cross-slip cases. Making the reasonable assumption that the nominal parameters of Table 1 provide a representative basis to define the structure of the variations, a Nelder-Mead optimisation of $F_{y}$ vs $\alpha$ is repeated over a full grid of $F_{z}, S$ to provide the surfaces illustrated in Figure 3.

\section{** INSERT FIGURE 3 **}

By examination of these, and equivalent maps for $F_{x}$, a series of candidate functions for $A, B$ and $b$ can be explored; Figure 3 also illustrates the quality of fit of two of these candidate functions, for $A$ and $b$ - the functions themselves are discussed below. Significantly, to maximise model efficiency, $F_{x}$ and $F_{y}$ will both be mapped using a single set of coefficients. As with other tyre models, to facilitate this a single additional scaling factor, $\eta$ is introduced, to scale longitudinal slip.

Table 2 details sets of candidate functions, chosen for their potential to map the vertical load and cross slip variations in $A, B$ and $b$ as simply as possible using a small number of parameters (f,g,h,p,q,r as required). $\gamma$ is the cross slip ( $\gamma=\eta S$ in the model for $F_{y}$ and $\gamma=\alpha$ in the model for $\left.F_{x}\right) . \bar{F}_{z}=F_{z} / 1000$ is used so that parameter magnitudes are of similar order. With six candidate functions in set 1 , each using any of four functions for $Z_{A}$ (set 2), there are 24 possible candidates for the model of $A$. In a similar way, sets 3 and 4 are coupled to provide six possible models for $B$, and sets 5 and 6 provide 9 possibilities for $b$.

\section{** INSERT TABLE 2 **}

Unfortunately, directly fitting each pair of sets of the candidate functions to the surfaces for $A$, $B$ and $b$ is not successful when the final model is tested in combination. This is because, while the quality of fit to $F_{x}, F_{y}$ is directly sensitive to most of the variations, there is a problem with poor conditioning in the model at high cross-slip values, which makes the high negative $B$ values at high $\gamma$ irrelevant (Figure 3). Considering Figure 2, it is clear that higher 
cross-slip cases result in force curves with ever slower exponential rise rate (lower $b$ at higher cross slip, are seen in Figure 3). This extends to the point where a constant $F_{y}$ or $F_{x}$ value is not attained within the useful range of slip. In these cases the second term in eqns (1), (2) becomes redundant, and the model could effectively collapse to $\bar{F}_{y}=A \alpha e^{-b \alpha}$. The large change in $B$ at higher $\gamma$ is thus a result of worsening conditioning of the parameter fit, so it should not be necessary for the model for $B$ to match the illustrated figure at these high cross slip values.

To establish the best combination of subfunctions without excessive reliance on the shapes of Figure 3, a factorial experiment was conducted to correlate quality of fit in the final $F_{x}, F_{y}$ model with all possible combinations of the subfunctions $(24 \times 6 \times 9=1296$ in all $)$. A weighted cost $J$ is applied,

$$
J=\sum_{\text {grid }} W_{x}\left(\bar{F}_{x r e f}-\bar{F}_{x}\right)^{2}+W_{y}\left(\bar{F}_{y r e f}-\bar{F}_{y}\right)^{2}
$$

With

$$
\begin{aligned}
& W_{x}=e^{-10 \bar{\alpha}} e^{-2 \bar{S}}\left(1-1 / 2\left|\bar{F}_{z}-\bar{F}_{z r}\right|\right)+\varepsilon \\
& W_{y}=e^{-10 \bar{S}} e^{-2 \bar{\alpha}}\left(1-1 / 2\left|\bar{F}_{z}-\bar{F}_{z r}\right|\right)+\varepsilon
\end{aligned}
$$

over a 3D grid of values spanning $1<\bar{F}_{z}<5,0<\bar{\alpha}<1,0<\bar{S}<1$. The weighting functions $W_{x}$ and $W_{y}$ are nominal in structure, designed to maximise quality of fit in the most critical lower slip and lower cross slip regions of the grid. $\bar{F}_{z r}=3$ increases the weighting around the rated tyre load of $3000 \mathrm{~N}$, and $\varepsilon=0.01$ ensures all grid positions have some influence. Precise setting of the weighting coefficients is not critical, but it is essential to provide significant bias to the fit around low (and hence also peak) slip regions, since the fitted grid necessarily covers a wide range of slip and load.

The resulting 1296 cost values were regressed against a set of 15 factors, coded at levels $-1,0$ or 1 according to the subfunctions selected. Table 3 summarises the coding of these factors.

\section{** INSERT TABLE $3 * *$}

All possible single factors and combinations of factors are then mapped to the normalised cost values by

$$
a_{1} \mathrm{f}_{1}+a_{2} \mathrm{f}_{2}+\cdots a_{15} \mathrm{f}_{15}+a_{16} \mathrm{f}_{1} \mathrm{f}_{2}+a_{17} \mathrm{f}_{1} \mathrm{f}_{3}+\cdots a_{1295} \prod_{i=1}^{15} \mathrm{f}_{i}=\frac{\bar{J}-J}{J_{\max }} \times 100
$$

where $\bar{J}$ is the mean cost and $J_{\max }=\max (\bar{J}-J)$. The coefficients $\underline{\theta}=\left[\begin{array}{llll}a_{1} & a_{2} & \cdots & a_{1295}\end{array}\right]^{T}$ are found by ordinary least squares solution of the problem $U \underline{\theta}=\underline{y}$, with $\underline{y}$ the vector of normalised cost values, and $U$ the matrix of factors for each model setting, with each row set according to eqn (23). In this way, the coefficients $a_{1}-a_{1295}$ provide significance values which quantify the importance of each factor across the variation of costs recorded. 
Figure 4 shows a normal score plot (see Kasperski \& Schneider, 1996) of the sorted $a_{i}$ values (scores); factors (or their combinations) which are insignificant are aligned with the straight line. Those outliers that have a significant positive or negative influence can be used to make a judgement on the best and worst choices. By examining the results in this way we do not simply select the combination of subfunctions which gives the lowest cost; we consider the average effect of each subfunction choice across all possible combinations, and can also determine which combinations of choices work best together. The final choice will then also need to take into account the relative complexity of the functions and number of parameter each subfunction requires.

\section{** INSERT FIGURE 4 **}

A selection of the scores with largest magnitude is annotated in Figure 4, and decoded in Table 4 to illustrate the process. Note that positive score values denote positive results (reduction in cost).

\section{** INSERT TABLE $4 * *$}

Considering these results, and with reference also to Table 3 , the first result, for $\mathrm{f}_{9}$ shows that cost is reduced by choosing the simplest model in set 3 . This reduces $\mathrm{B}$ to dependence on load alone, and is pertinent to the earlier discussion of conditioning; by using this simple function we prevent the final model from suffering from poor conditioning. The remaining advice in Table 5 can be followed to further narrow the field of choices to :

set 1 : (iii)

set 4 : (ii) or (iii) set 2 : (ii), (iii) or (iv) set $5:$ (i) set 3 : (i)

set 6 : choice not significant

It is interesting that a relatively complex load model is required in set 4 . Separate experiments confirm significantly poorer results when this load dependence is simplified, and further examination of other factor scores leads to the choice of function (ii) rather than (iii) here.

Finally, taking the complexity of subfunctions and the number of coefficients each needs into account, it is clear that set 6 should use subfunction (i), since this introduces no additional coefficients and reduces the model for $b$ to dependence on cross slip alone. For set 2, subfunction (iii) can be ignored, as this requires more coefficients than the alternatives. This leaves 2 possible variants for set 2, which can not be decided using normal scoring and model complexity, so a final choice of set 2 = (iv) was decided purely on the basis of lowest cost.

Given the six subfunction choices, and making some straightforward parameter name substitutions, the final model is

$$
\begin{gathered}
\bar{S}=\eta|S| \quad \bar{\alpha}=|\alpha| \\
A_{x}=A_{1} e^{A_{2} \bar{F}_{z}} e^{-A_{3} \bar{\alpha}}+A_{4} \bar{\alpha} \quad A_{y}=A_{1} e^{A_{2} \bar{F}_{z}} e^{-A_{3} \bar{S}}+A_{4} \bar{S} \\
B=B_{1}+B_{2} e^{-B_{3} \bar{F}_{z}}
\end{gathered}
$$




$$
\begin{array}{cc}
b_{x}=b_{1} e^{-b_{2} \bar{\alpha}} & b_{y}=b_{1} e^{-b_{2} \bar{S}} \\
\bar{F}_{x}=A_{x} \bar{S} e^{-b_{x} \bar{S}}+B\left(1-e^{-b_{x} \bar{S}}\right) & \bar{F}_{y}=A_{y} \bar{\alpha} e^{-b_{y} \bar{\alpha}}+B\left(1-e^{-b_{y} \bar{\alpha}}\right) \\
F_{x}=\mu F_{z} \bar{F}_{x} \operatorname{sgn}(S) & F_{y}=\mu F_{z} \bar{F}_{y} \operatorname{sgn}(\alpha)
\end{array}
$$

Which employs 10 parameters; $A_{1}-A_{4}, B_{1}-B_{3}, b_{1}, b_{2}, \eta$.

With the full model structure now defined, parameter sets can be identified to match tyre data whenever a new tyre is required. This can be done by Nelder Mead (Matlab fminsearch) optimisation on $F_{x}, F_{y}$ data at varying slip, cross slip and vertical load conditions, either measured or from a reference model. Figure 5 shows how the new model performs when fitted to the reference model (Table 1) across the full range of $F_{x}$ and $F_{y}$ for varying slip and cross-slip, at three vertical loads spanning the most relevant range. There is some compromise in the solution, most notably in peak force at zero cross-slip, but considered across the whole map, the accuracy is very good.

\section{** INSERT FIGURE 5 **}

\section{Computational Advantage}

The significant appeal of the new model is its computational advantage and simplicity in force calculations, and more particularly in the computation of derivatives. The full model Jacobian is required in applications such as the extended Kalman Filter, or any gradient-based control optimisation, so $\partial F_{y} / \partial \alpha, \partial F_{y} / \partial S, \partial F_{y} / \partial F_{z}, \partial F_{x} / \partial \alpha, \partial F_{x} / \partial S, \partial F_{x} / \partial F_{z}$ may all be required. With the Pacejka model, the combination of the magic formula itself and the total slip $k=\sqrt{\bar{S}^{2}+\bar{\alpha}^{2}}$ makes all these derivatives prohibitively complicated. One would not attempt to compute them by hand, but even using an algebraic tool such as Maple or Matlab's symbolic toolbox, the expressions for the Pacejka derivatives are so long that they cannot even be cut and pasted into other codes. The chain rule can be used; eg

$$
\frac{\partial F_{y}}{\partial \alpha}=\frac{\partial F_{y}}{\partial F_{r}} \frac{\partial F_{r}}{\partial k} \frac{\partial k}{\partial \alpha}+\frac{\partial F_{y}\left(F_{r}\right)}{\partial \alpha}
$$

but even with this short cut, Table 5 shows the scale of the problem for the typical example $\partial F_{y} / \partial \alpha$; here the derivative has been computed and simplified using the Matlab symbolic toolbox, and the number of characters in the resulting expression is given.

$$
\text { ** INSERT TABLE } 5 \text { ** }
$$

Unlike the alternatives, all six of the Exponential model's derivatives can be written algebraically. For this example : 


$$
\frac{\partial F_{y}}{\partial \alpha}=\frac{\mu F_{z}}{e^{\eta b_{2}|S|}}\left(B_{1} b_{1}+\eta A_{4}|S|\left(e^{\eta b_{2}|S|}-|\alpha| b_{1}\right)+\frac{A_{1}\left(e^{\eta b_{2}|S|}-b_{1}|\alpha|\right)}{e^{A_{2} F_{z}} e^{\eta A_{3}|S|}}+\frac{B_{2} b_{1}}{e^{B_{3} F_{z}}}\right) / e^{b_{1}|\alpha| e^{\eta b_{2}|S|}}
$$

For further comparison, a Dugoff model is also compared here. Another alternative and simple combined slip model, this has been taken from Ding and Taheri, 2010; it generates more manageable derivative expressions, and relies on just four parameters. Unfortunately, in a free optimisation to the reference model, it is not sufficiently accurate; this is illustrated in Figure 6, which is directly comparable to Figure 5.

As a further demonstration of computational advantage, all three models were used to evaluate $F_{x}, F_{y}$ and separately, $\partial F_{y} / \partial \alpha$ for 10,000 randomly chosen input values. The time taken to complete those computations, averaged over 10 trials, on an Intel $67002.66 \mathrm{GHz}$ processor with 4GB RAM, is given in Table 6. In both force and derivative cases the exponential model is very much faster, and in derivative calculations it is two orders of magnitude faster than the reference.

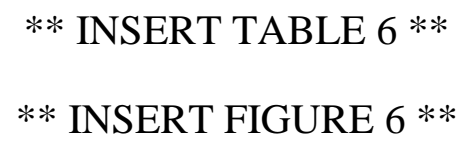

\section{Fitting to Measured Tyre Data}

As a further confirmation of the Exponential model's suitability and accuracy, measured tyre data is mapped. Such data is notoriously difficult to acquire, and a full combined slip mapping was not available here. However, single slip characteristics for $F_{x}$ vs $S$ and $F_{y}$ vs $\alpha$ for the same tyre, at three different vertical loads were sourced from the Formula SAE Tire Test Consortium, and with thanks to this consortium and the Calspan Tire Research Facility, these are illustrated in Figure 7.

\section{** INSERT FIGURE 7 **}

In the absence of cross-slip data, eqn (25) reduces to

$$
A_{x}=A_{1} e^{A_{2} \bar{F}_{z}} \quad A_{y}=A_{1} e^{A_{2} \bar{F}_{z}}
$$

and eqn (27) reduces to

$$
b_{x}=b_{y}=b_{1}
$$

The fit is very good, although some loss of accuracy is seen in longitudinal stiffness at the higher loads. Here, an equal weighting, $W_{x}=W_{y}=1$ has been applied at all slip values; it is easy to alter the quality of fit at higher vs lower slip values by adjusting the weightings in an obvious way.

Given the Exponential model structure, a fully combined slip version of this tyre data can be constructed by carrying over the cross-slip variation parameters, $A_{3}, A_{4}$ and $b_{2}$ from another fit. Figure 8 shows the resulting model of $F_{x}$, using cross-slip variations from the fit to the 
reference model carried out earlier; the result is demonstrably accurate for both $F_{x}$ and $F_{y}$ under pure slip conditions, while also providing an appropriate, if nominal variation over all combined slip combinations.

\section{** INSERT FIGURE 8 **}

\section{Conclusion}

In its simplest form the Exponential tyre model describes single slip tyre data effectively using just three parameters; these can be matched to required stiffness, peak and saturated force criteria. The combined slip variant has been developed through rigorous statistical analysis of candidate functions, and can accurately map the results of a proven reference model. The model structure is simple, being fully described by four short functions using 10 parameters. It can be fitted to measured tyre data, and is also a useful complement to existing tyre models; after fitting the Exponential model to an existing more complicated model, the Exponential model can replace its complex counterpart in computationally exhaustive or realtime applications. The simple model structure can also be adapted to fit available data if the full map of force variation across slips and load is not available. This has been demonstrated, with good fits to measured data resulting in a case where only single slip data was available.

\section{References}

Andreasson, J. \& Bunte, T. (2006) "Global chassis control based on inverse vehicle dynamics models”, Vehicle System Dynamics, v44, nSUPPL.1, p321-328.

Best, M.C. (2010) "Exploring assumptions and requirements for continuous modification of vehicle handling using nonlinear optimal control and a new exponential tyre model", Proceedings of the $10^{\text {th }}$ International Symposium on Advanced Vehicle Control (AVEC), Loughborough, UK, August 2010, p79 - 84.

Ding, N., \& Taheri, S. (2010) “A modified Dugoff tire model for combined-slip forces”, Tire Science and Technology, v38, n3, p228-244.

Doumiati, M., Victorino, A. C., Charara, A. \& Lechner, D. (2011) "Onboard real-time estimation of vehicle lateral tire-road forces and sideslip angle", IEEE/ASME Transactions on Mechatronics, v16, n4, p601-614

Gordon, T.J. \& Best, M.C. (2006) "On the synthesis of driver inputs for the simulation of closed-loop handling manoeuvres”, International Journal of Vehicle Design v40, n1/2/3 p5276.

Kasperski, W.J. \& Schneider, H. (1996) "Using normal probability plots to find significant factors", Proceedings of the Annual Meeting of the Decision Sciences Institute, v2, p11721174. 
Kim, J. (2009) "Identification of lateral tyre force dynamics using an extended Kalman filter from experimental road test data”, Control Engineering Practice, v17, n3, p 357-367.

Li, D., Du, S. \& Yu, F. (2008) "Integrated vehicle chassis control based on direct yaw moment, active steering and active stabiliser”, Vehicle System Dynamics, v46, nSUPPL.1, p341-351.

Milliken, D.L. and Milliken, W.F. (1995) Race Car Vehicle Dynamics (3rd ed.) SAE International.

Pacejka, H.B. (2002) Tyre and Vehicle Dynamics (1st ed.) Butterworth Heinemann.

Poussot-Vassal, C., Sename, O. \& Dugard, L. (2008) “A LPV/Hœ global chassis controller for handling improvements involving braking and steering systems”, Proceedings of the 47th IEEE Conference on Decision and Control, (CDC), p5366-5371.

Wenzel, T.A., Burnham, K.J., Blundell, M.V. \& Williams, R.A. (2006) "Dual extended Kalman filter for vehicle state and parameter estimation”, Vehicle System Dynamics, v44, n2, p153-171. 


\section{Tables}

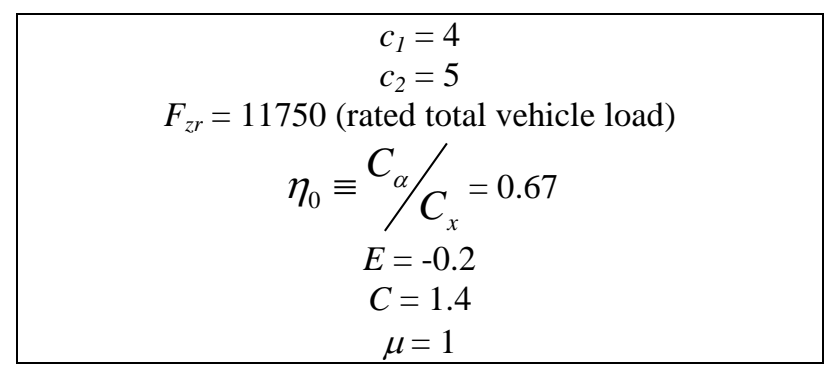

Table 1 : Reference Model Tyre Parameters

\begin{tabular}{|l|l|l|}
\hline \multicolumn{1}{|c|}{ set 1: functions for $A$} & \multicolumn{1}{|c|}{ set 3: functions for $B$} & \multicolumn{1}{|c|}{ set 5: functions for $b$} \\
\hline (i) $Z_{A} e^{-f \gamma}$ & (i) $Z_{B} f$ & (i) $Z_{b} e^{-f \gamma}$ \\
(ii) $Z_{A} e^{-f \gamma}+g$ & (ii) $Z_{B}\left(f-e^{g(\gamma-h)}\right)$ & (ii) $Z_{b}(f-g \gamma)$ \\
(iii) $Z_{A} e^{-f \gamma}+g \gamma$ & (iii) $Z_{b}\left(f \gamma^{2}-g \gamma+h\right)$ \\
(iv) $Z_{A} e^{-f \gamma}+g \gamma^{2}$ & \\
(v) $Z_{A} f /(g+\gamma)$ & \\
(vi) $Z_{A}\left(f \gamma^{2}-g \gamma+h\right)$ & & \\
\hline set $2:$ load functions in $A$ & set 4 : load functions in $B$ & set 6 : load functions in $b$ \\
\hline (i) $Z_{A}=p$ & (i) $Z_{B}=p$ & (i) $Z_{b}=p$ \\
(ii) $Z_{A}=p-q \bar{F}_{z}$ & (ii) $Z_{B}=p+q e^{-r \bar{F}_{z}}$ & (ii) $Z_{b}=p e^{-q \bar{F}_{z}}$ \\
(iii) $Z_{A}=p \bar{F}_{z}^{2}-q \bar{F}_{z}+r$ & (iii) $Z_{B}=p-q \bar{F}_{z}$ & (iii) $Z_{b}=p-q \bar{F}_{z}$ \\
(iv) $Z_{A}=p e^{-q \bar{F}_{z}}$ & & \\
\hline
\end{tabular}

Table 2 : Candidate cross slip and load dependence functions for $A, B$ and $b$

\begin{tabular}{|c|c|c|c|}
\hline factor & \multicolumn{3}{|l|}{ setting } \\
\hline $\mathrm{f}_{1}$ & $=1$ if set $1 \mathrm{fn}=(\mathrm{i})$ & \multicolumn{2}{|l|}{$=0$ otherwise } \\
\hline $\mathrm{f}_{2}$ & $=1$ if set $1 \mathrm{fn}=(\mathrm{ii})$ & \multicolumn{2}{|l|}{$=0$ otherwise } \\
\hline $\mathrm{f}_{3}$ & $=1$ if set $1 \mathrm{fn}=($ iii) & \multicolumn{2}{|l|}{$=0$ otherwise } \\
\hline $\mathrm{f}_{4}$ & $=1$ if set $1 \mathrm{fn}=(\mathrm{iv})$ & \multicolumn{2}{|l|}{$=0$ otherwise } \\
\hline $\mathrm{f}_{5}$ & $=1$ if set $1 \mathrm{fn}=(\mathrm{v})$ & $=-1$ if set $1 \mathrm{fn}=(\mathrm{vi})$ & $=0$ otherwise \\
\hline $\mathrm{f}_{6}$ & $=1$ if set $2 \mathrm{fn}=(\mathrm{i})$ & \multicolumn{2}{|l|}{$=0$ otherwise } \\
\hline $\mathrm{f}_{7}$ & $=1$ if set $2 \mathrm{fn}=(\mathrm{ii})$ & \multicolumn{2}{|l|}{$=0$ otherwise } \\
\hline $\mathrm{f}_{8}$ & $=1$ if set $2 \mathrm{fn}=($ iii) & $=-1$ if set $2 \mathrm{fn}=(\mathrm{iv})$ & $=0$ otherwise \\
\hline $\mathrm{f}_{9}$ & $=1$ if set $3 \mathrm{fn}=(\mathrm{i})$ & \multicolumn{2}{|l|}{$=-1$ if set $3 \mathrm{fn}=(\mathrm{ii})$} \\
\hline$f_{10}$ & $=1$ if set $4 \mathrm{fn}=(\mathrm{i})$ & \multicolumn{2}{|l|}{$=0$ otherwise } \\
\hline$f_{11}$ & $=1$ if set $4 \mathrm{fn}=(\mathrm{ii})$ & $=-1$ if set $4 \mathrm{fn}=(\mathrm{iii})$ & $=0$ otherwise \\
\hline$f_{12}$ & $=1$ if set $5 \mathrm{fn}=(\mathrm{i})$ & \multicolumn{2}{|l|}{$=0$ otherwise } \\
\hline$f_{13}$ & $=1$ if set $5 \mathrm{fn}=(\mathrm{ii})$ & $=-1$ if set $5 \mathrm{fn}=(\mathrm{iii})$ & $=0$ otherwise \\
\hline$f_{14}$ & $=1$ if set $6 \mathrm{fn}=(\mathrm{i})$ & \multicolumn{2}{|l|}{$=0$ otherwise } \\
\hline $\mathrm{f}_{15}$ & $=1$ if set $6 \mathrm{fn}=(\mathrm{ii})$ & $=-1$ if set $6 \mathrm{fn}=(\mathrm{iii})$ & $=0$ otherwise \\
\hline
\end{tabular}

Table 3 : Relationship between factors and subfunction selection 


\begin{tabular}{|c|c|c|c|}
\hline annot $^{n}$ & $a$ & factor comb ${ }^{n}$ & interpretation from factor definitions \\
\hline I & +9.9 & $f_{9}$ & do use set $3=$ (i) rather than set $3=$ (ii) \\
\hline II & +9.3 & $\mathrm{f}_{3} \mathrm{f}_{12}$ & do use set $1=$ (iii) in combination with set $5=$ (i) \\
\hline III & +8.3 & $\mathrm{f}_{12}$ & do use set $5=(\mathrm{i})$ \\
\hline IV & +6.2 & $\mathrm{f}_{3}$ & do use set $1=$ (iii) \\
\hline $\mathrm{V}$ & +5.6 & $\mathrm{f}_{4}$ & do use set $1=(\mathrm{iv})$ \\
\hline $\mathrm{I}$ & -17.6 & $\mathrm{f}_{6} \mathrm{f}_{10}$ & don't use set $2=$ (i) in combination with set $4=$ (i) \\
\hline II & -9.8 & $\mathrm{f}_{10}$ & don’t use set 4 = (i) \\
\hline III & -6.7 & $\mathrm{f}_{2}$ & don't use set $1=$ (ii) \\
\hline IV & -6.5 & $\mathrm{f}_{6}$ & don’t use set $2=(\mathrm{i})$ \\
\hline $\mathrm{V}$ & -6.1 & $\mathrm{f}_{13}$ & don't use set $5=$ (ii) and / or do use set $5=$ (iii) \\
\hline
\end{tabular}

Table 4 : Selected information decoded from the normal score plot

\begin{tabular}{|c|c|}
\hline$\partial F_{y} / \partial \alpha$ & $\begin{array}{c}\text { Number of } \\
\text { characters }\end{array}$ \\
\hline Reference model; direct derivative expression & 5904 \\
Reference model; evaluation using eqn (28) & 4068 \\
Dugoff model & 833 \\
Exponential model & 218 \\
\hline
\end{tabular}

Table 5 : Size of derivative functions

\begin{tabular}{|c|c|c|}
\hline Time for 10,000 executions (seconds) & $F_{y} \& F_{x}$ & $\partial F_{y} / \partial \alpha$ \\
\hline Reference model & 0.127 & 2.140 \\
Dugoff model & 0.077 & 0.118 \\
Exponential model & 0.038 & 0.028 \\
\hline
\end{tabular}

Table 6 : Speed of execution of tyre models

\section{Figure Captions}

Figure 1 : Components of the Single Slip Exponential Tyre Model

Figure 2 : Single slip Exponential tyre model fitted to the reference model

Figure $3: F_{y}$ model coefficient variation with load and cross-slip, with fitted potential candidate models for $A$ and $b$

Figure 4 : Normal score plot of factors affecting Exponential model fit quality

Figure 5 : Combined slip Exponential tyre model fitted to the reference model

Figure 6 : Combined slip Dugoff tyre model fitted to the reference model

Figure 7 : Exponential model fitted to the raw measured tyre forces

Figure 8 : Expanding the Exponential model to provide an appropriate combined slip map 


\section{Figures}

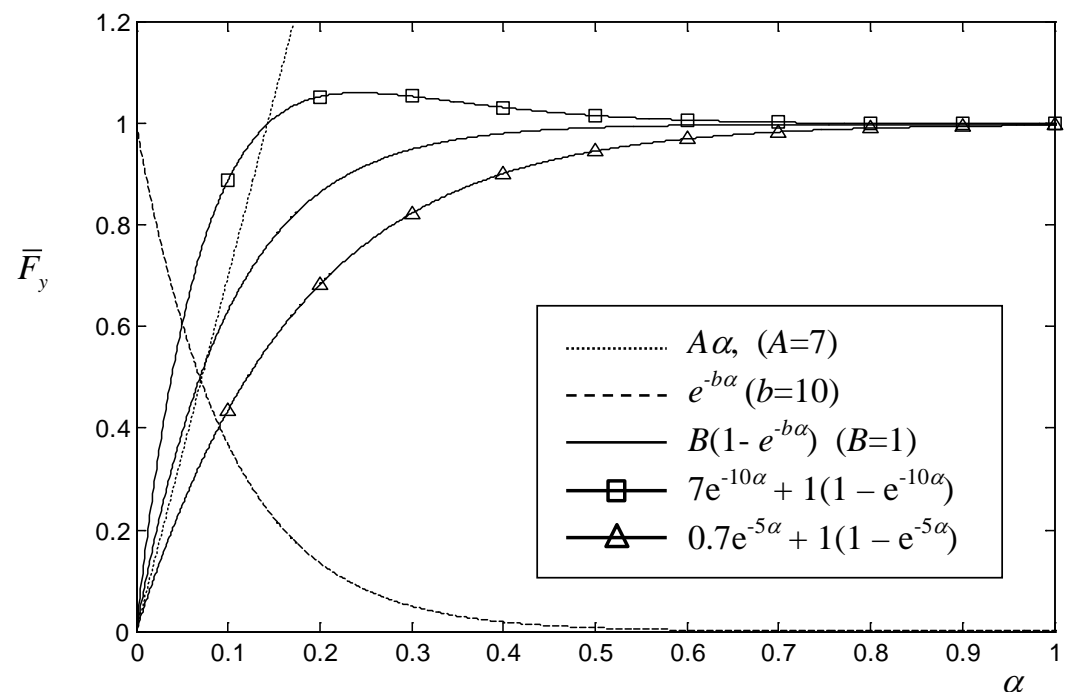

Figure 1 : Components of the Single Slip Exponential Tyre Model

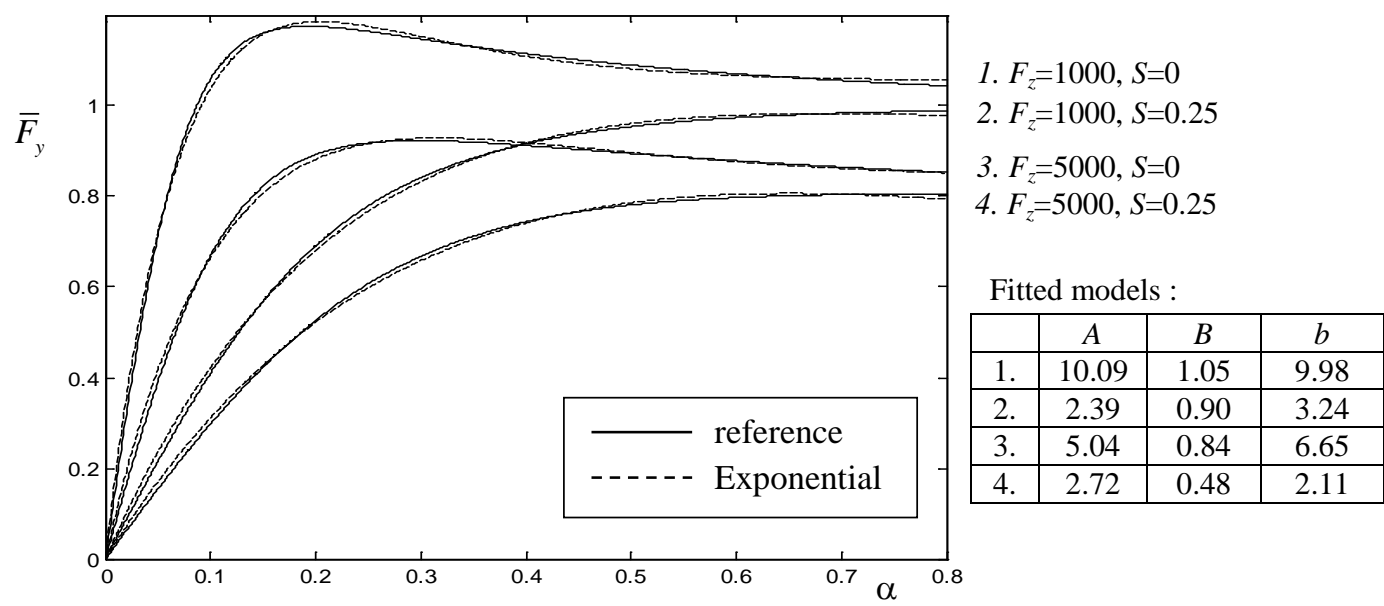

Figure 2 : Single slip Exponential tyre model fitted to the reference model 

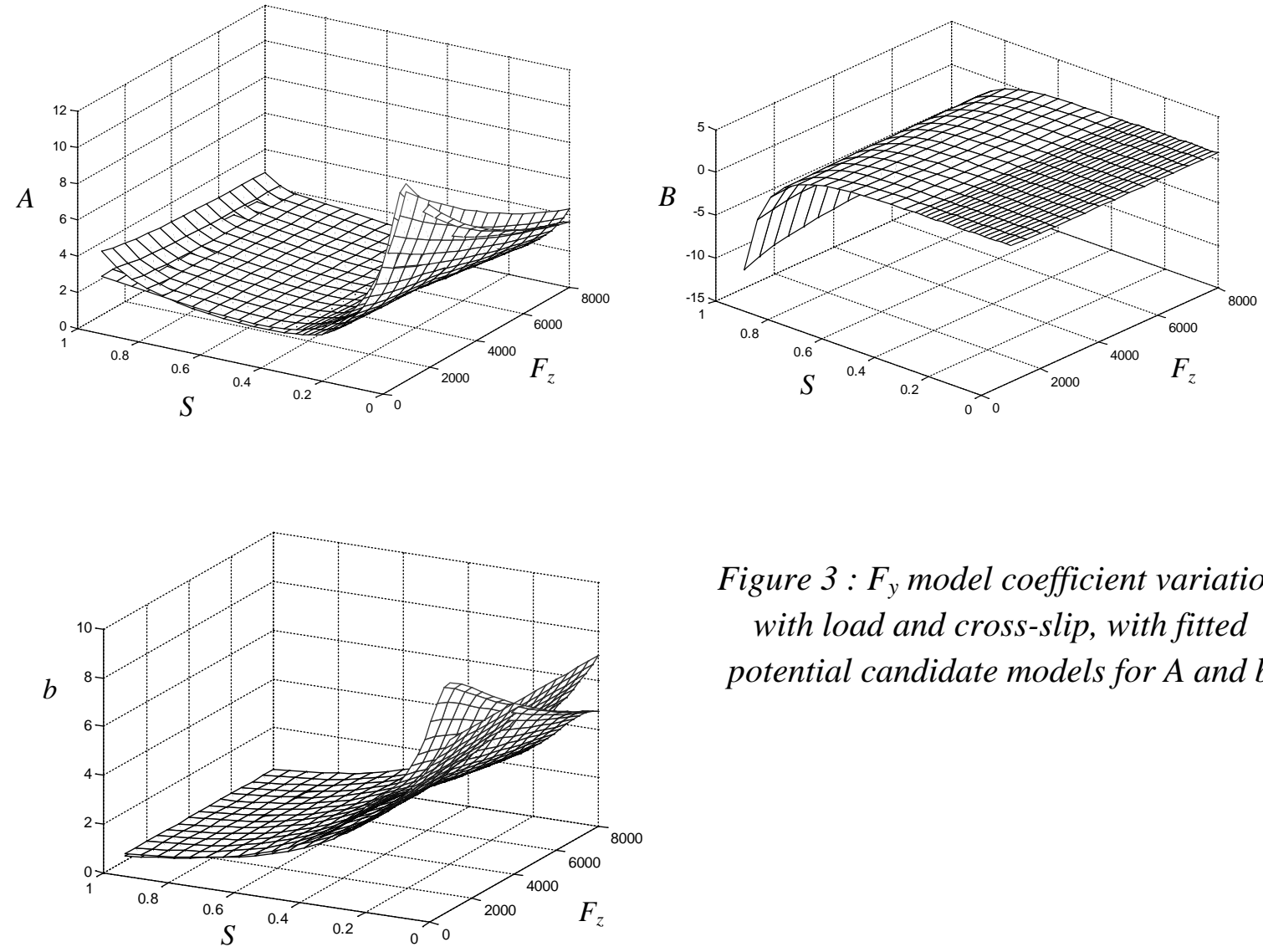

Figure 3 : $F_{y}$ model coefficient variation with load and cross-slip, with fitted potential candidate models for $A$ and $b$

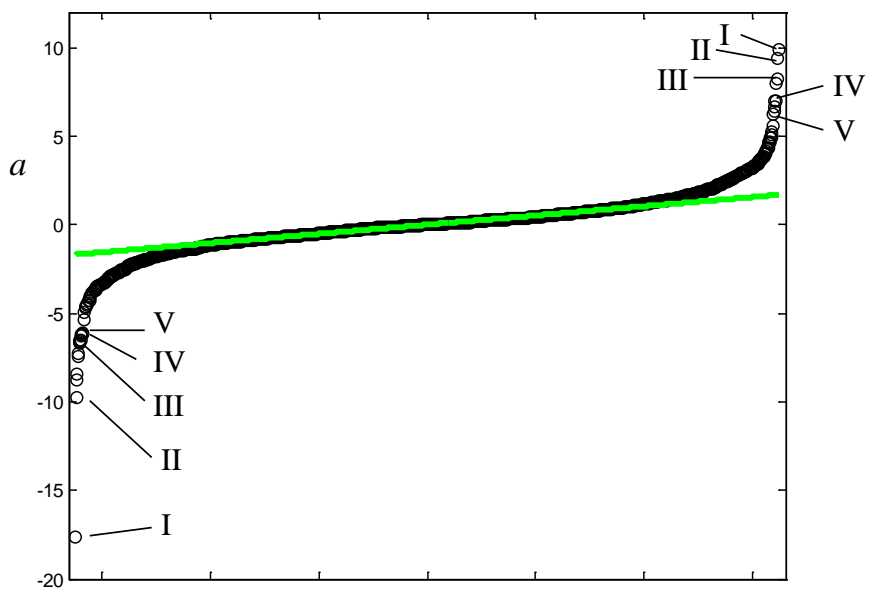

Figure 4 : Normal score plot of factors affecting Exponential model fit quality 
top row, $F_{z}=1000 \mathrm{~N}$
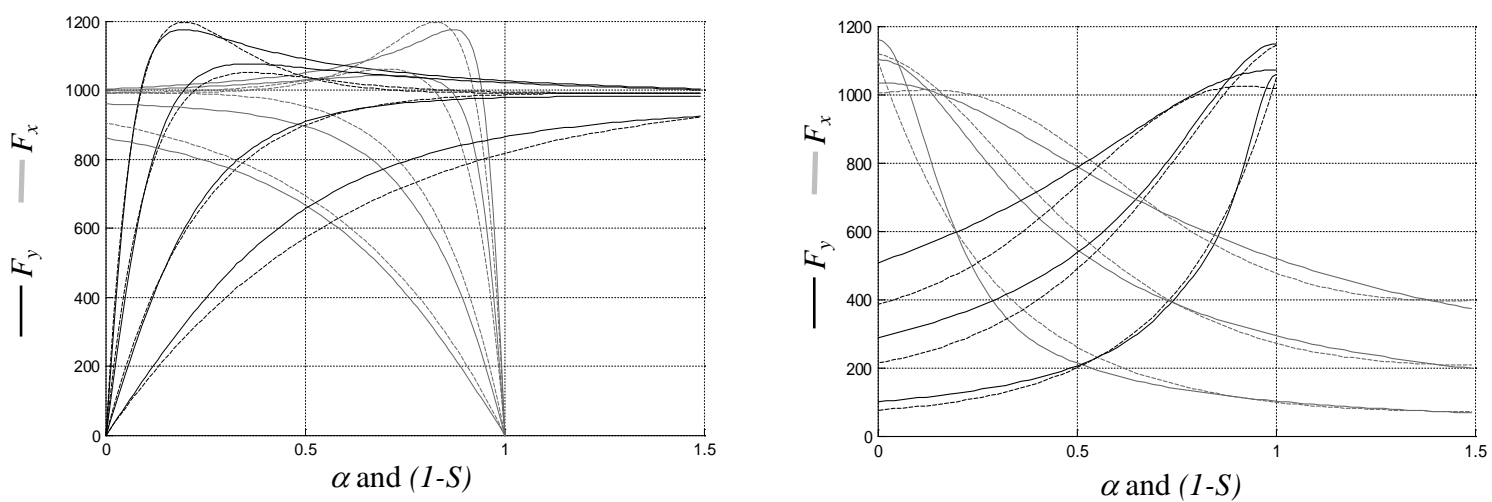

middle row, $F_{z}=3000 \mathrm{~N}$
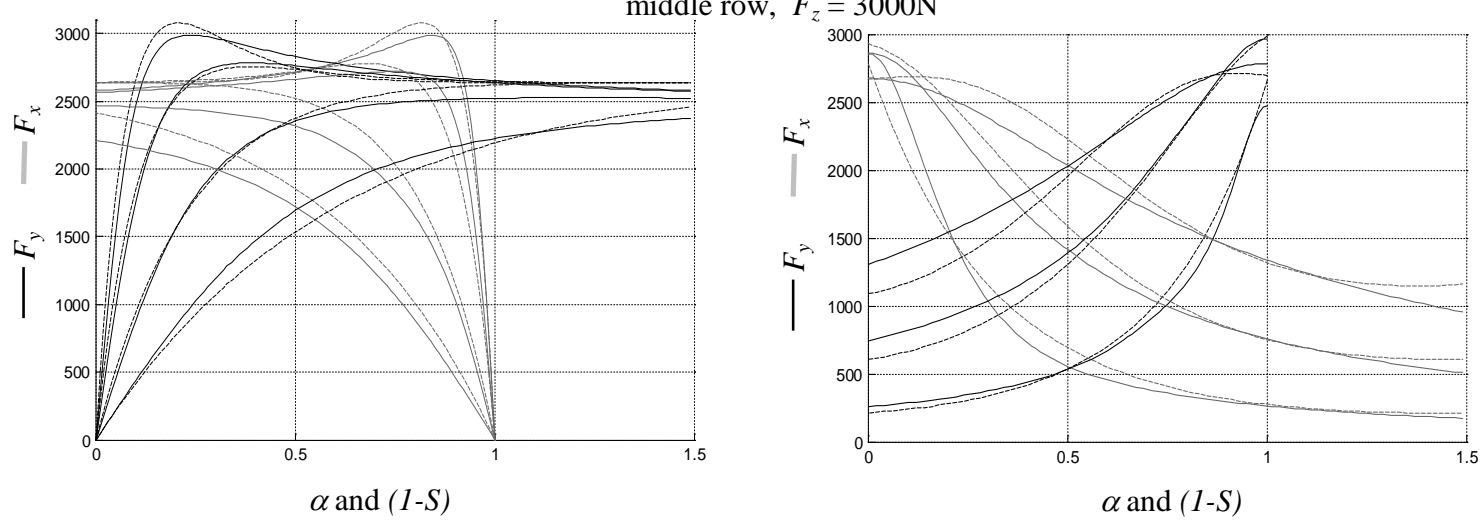

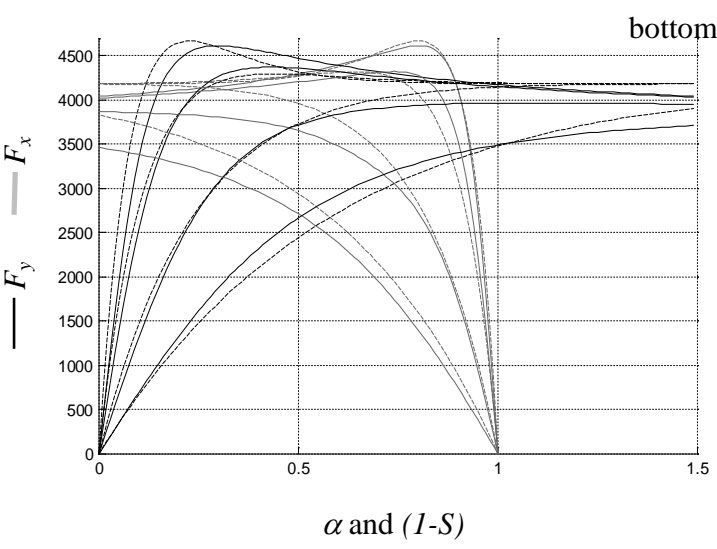

Left hand plots show $F$ vs slip solid $=$ reference, dashed $=$ Exponential Exponential model parameters :

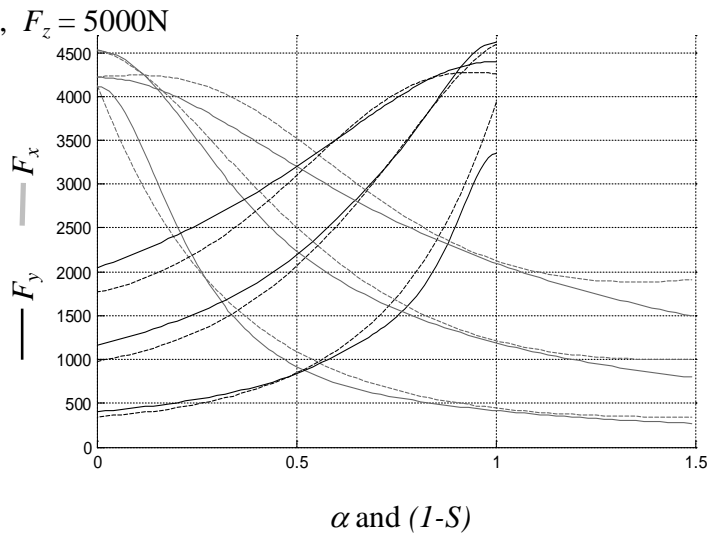

Right hand plots show $F$ vs cross slip solid $=$ reference, dashed $=$ Exponential solid

$A_{1}=12.828 \quad A_{2}=0.118 \quad A_{3}=8.057 \quad A_{4}=0.329 \quad B_{1}=0.811 \quad B_{2}=0.292 \quad B_{3}=0.486 \quad b_{1}=9.164 \quad b_{2}=2.746 \quad \eta=1.129$

Figure 5 : Combined slip Exponential tyre model fitted to the reference model 


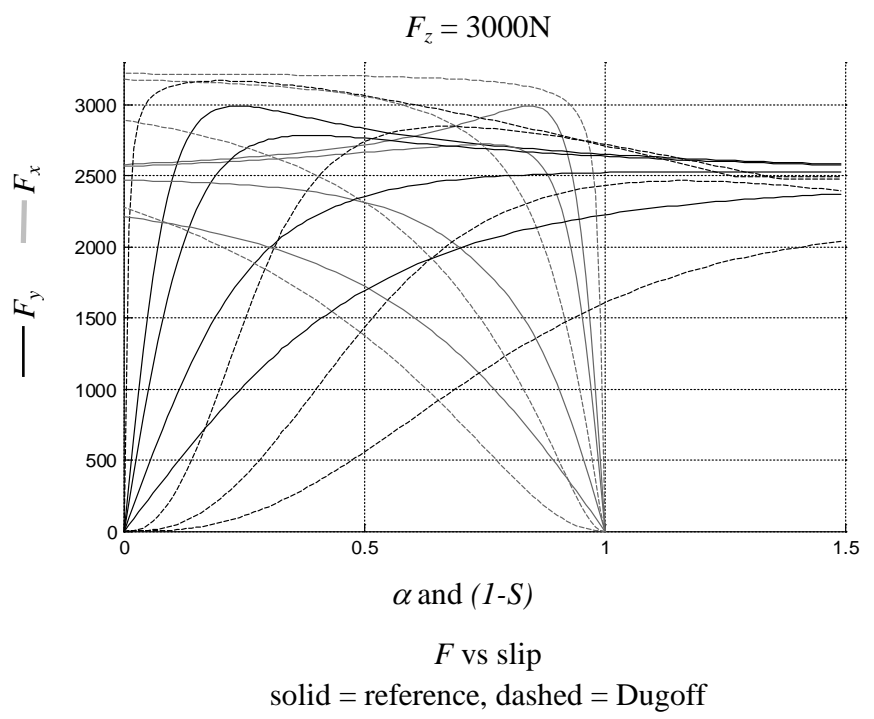

Figure 6 : Combined slip Dugoff tyre model fitted to the reference model

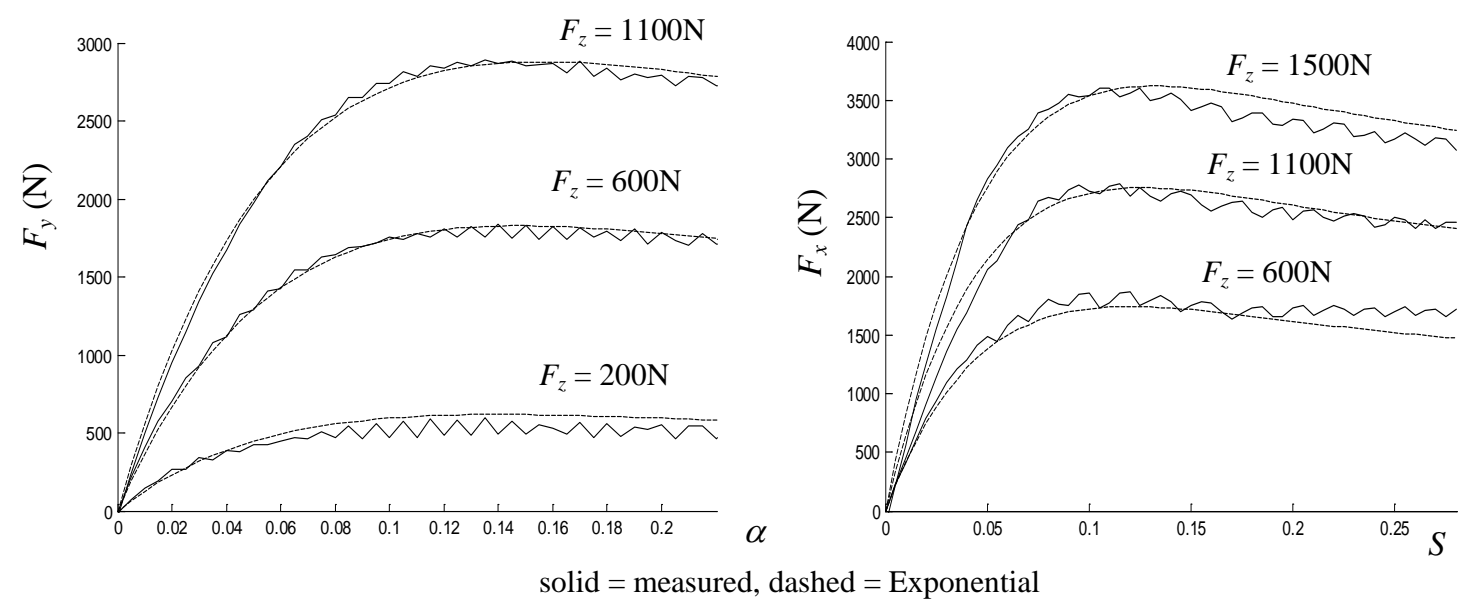

Figure 7 : Exponential model fitted to the raw measured tyre forces 


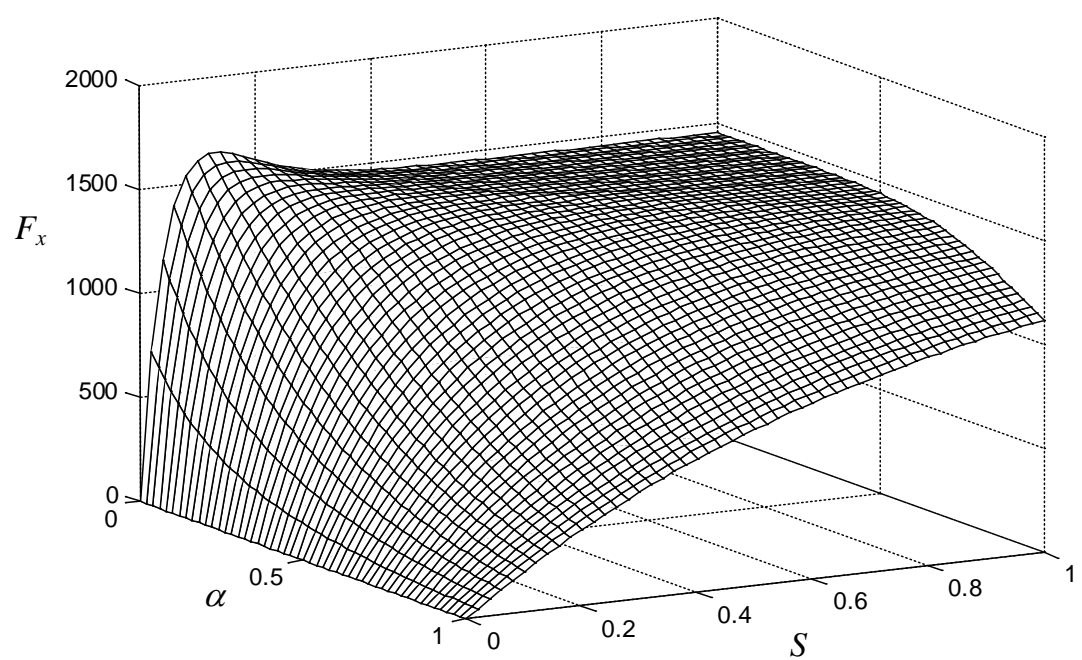

Exponential model parameters :

$A_{1}=32.10 \quad A_{2}=0.051 \quad A_{3}=7.711 \quad A_{4}=0.290 \quad B_{1}=2.507 \quad B_{2}=-0.025 \quad B_{3}=-2.008 \quad b_{1}=13.428 \quad b_{2}=2.485 \quad \eta=1.170$

Figure 8 : Expanding the Exponential model to provide an appropriate combined slip map 